\title{
When should the elephant (frozen elephant trunk) enter the room (aorta)?
}

\author{
Frank A. Baciewicz, Jr, MD
}

\footnotetext{
From the Cardiothoracic Surgery Section, Michael and Marian Ilitch Department of Surgery, Wayne State University School of Medicine, Detroit, Mich.

Disclosures: Author has nothing to disclose with regard to commercial support.

Received for publication April 24, 2017; accepted for publication May 5, 2017; available ahead of print June 27, 2017.

Address for reprints: Frank A. Baciewicz, Jr, MD, Harper Hospital, 3990 John R St, Detroit, MI 48201 (E-mail: fbaciewi@dmc.org).

J Thorac Cardiovasc Surg 2017;154:1190-1

$0022-5223 / \$ 36.00$

Copyright $(2) 2017$ by The American Association for Thoracic Surgery

http://dx.doi.org/10.1016/j.jtcvs.2017.05.037
}

In this issue of the Journal, Ma and colleagues ${ }^{1}$ have presented outstanding operative results in type A aortic dissection in patients with Marfan syndrome, with an in-hospital mortality of $6.0 \%$, a 5 -year survival of $86 \%$, and a reoperation rate of $12 \%$ at 5 years. All patients were treated with total arch replacement (TAR) and frozen elephant trunk (FET).

Contemporary intervention for acute type A dissection shows the pendulum swinging to more extensive procedures including hemiarch or TAR with FET, particularly when the thoracic aorta is dilated or malperfusion is present. Publications detailing the repair of acute type A aortic dissection with FET by Katayama and colleagues, ${ }^{2}$ Shrestha and colleagues, ${ }^{3}$ and Kobayashi and colleagues ${ }^{4}$ have reported in-hospital mortality of $6 \%$, $12 \%$, and $14 \%$; 5-year survival of $75 \%, 80 \%$, and $70 \%$, and late reoperation rates of $7 \%, 12 \%$, and $15 \%$, respectively.

Based on the series reported by Rylski and coworkers, ${ }^{5}$ $30 \%$ of acute type A dissections in patients with Marfan syndrome will require secondary thoracic aortic intervention by 8 years postsurgery. In Marfan syndrome, placing an FET is controversial, given the descending thoracic aorta's abnormal connective tissue. The fact that Coselli and colleagues ${ }^{6}$ demonstrated excellent $4 \%$ mortality for patients with Marfan syndrome requiring reintervention on the descending thoracic aorta strengthens the argument against the use of FET in these patients.

Before the recommendations of $\mathrm{Ma}$ and colleagues are adopted, it must be recognized that their study population was a selective subgroup of patients with Marfan syndrome experiencing type A aortic dissection. The study included both acute $(n=40)$ and chronic $(n=66)$ subgroups, with acute defined as dissection occurring $<14$ days after diagnosis. The patients in the acute subgroup underwent surgery at a mean of $5.8 \pm 3.9$ days after the onset of symptoms; the chronic subgroup, at a mean of 62 days after symptom onset. Two of the 40 patients in the acute subgroup had undergone a previous cardiac procedure,

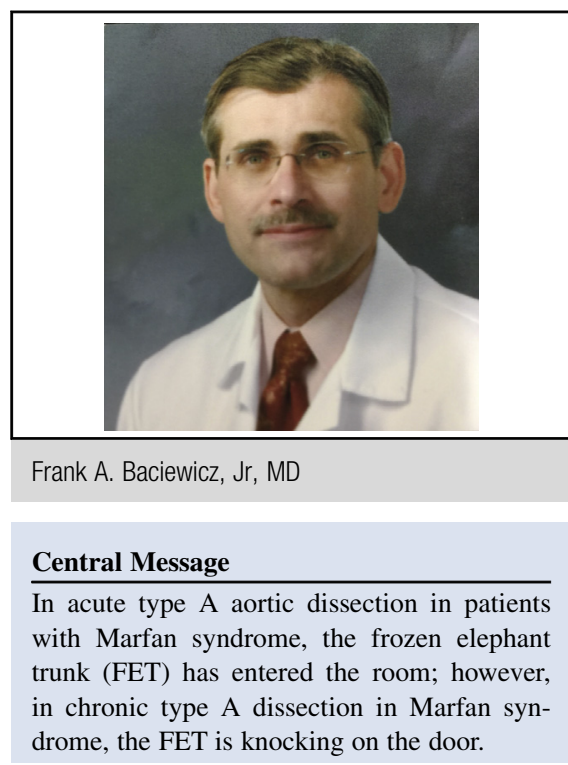

See Article page 1175 .

including 1 composite root replacement. In the chronic subgroup, 21 of 66 patients had undergone a previous cardiac procedure, including 15 root replacements, 2 ascending aortic replacements, 3 thoracic endovascular repairs, 1 aortic root wrapping, and 2 mitral operations. Eligibility criteria for TAR with FET included hemodynamic stability, no morbid malperfusion, dissection involving the ascending arch and descending aorta or limited to the ascending aorta, and a proximal descending aorta $>40 \mathrm{~mm}$ in diameter. Given the foregoing stipulations, the 106 reported Marfan patients with type A dissection treated with TAR with FET comprise slightly more than half the total 187 Marfan syndrome patients with type A dissection referred during the study period.

The FET is placed to prevent descending thoracic dilatation and ensure perfusion through the true lumen. Nevertheless, 6 patients died of multiorgan failure during the course of hospitalization, possibly related to acute malperfusion (none had malperfusion preoperatively), and 1 patient had abdominal aortic dissection (chronic group). Four patients had radiologic evidence of the FET perfusing the false lumen long-term. During long-term follow-up, 8 patients required thorocoabdominal aortic replacement, 3 required a thoracic stent for new thoracic entry tears, and 8 died from distal aortic rupture. In addition, the cause of death was unknown in 8 patients. Seven 
of the 11 interventions were performed in the chronic subgroup, 7 of the 8 patients with aortic rupture, and 6 of the 8 patients with an unknown cause of death, were in the chronic subgroup.

These results question whether FET should be placed in the descending thoracic aorta in the chronic subgroup. The reinterventions, deaths from rupture, and deaths from unknown causes in these patients make for less than a ringing endorsement of the use of FET in the chronic subgroup. Should the authors tailor their approach and favor TAR and FET in all patients with acute type A dissection, especially those with preoperative malperfusion? Should the descending thoracic aorta be fenestrated in patients with Marfan syndrome and chronic type A aortic dissection?

Shrestha and coworkers ${ }^{7}$ reported that patients with acute type A aortic dissection required significantly less aortic intervention $(8 \%)$ after TAR and FET, whereas $22 \%$ of the entire group required aortic intervention. The current consensus leans toward a more extensive operation in acute type A aortic dissection. In patients with Marfan syndrome with acute type A dissection, TAR and FET offer a reasonable approach, given the expectation for further thoracic aortic intervention. In chronic type A aortic dissection in Marfan syndrome, the use of FET awaits confirmation.

\section{References}

1. Ma W-G, Zhang W, Zhu J-M, Ziganshin BA, Zhi A-H, Zheng J, et al. Long-term outcomes of frozen elephant trunk for type A aortic dissection in patients with Marfan syndrome. J Thorac Cardiovasc Surg. 2017;154:1175-91.

2. Katayama A, Uchida N, Katayama K, Arakawa M, Sueda T. The frozen elephant trunk technique for acute type A aortic dissection: results from 15 years of experience. Eur J Cardiothorac Surg. 2015;47:355-60; discussion 360.

3. Shrestha M, Haverich A, Martens A. Total aortic arch replacement with the frozen elephant trunk procedure in acute DeBakey type I aortic dissection. Eur J Cardiothorac Surg. 2017;51(Suppl 1):i29-34.

4. Kobayashi M, Chaykovska L, van der Loo B, Nguyen TD, Puippe G, Salzberg S et al. Long-term results of simplified frozen elephant trunk technique in complicated acute type A aortic dissection: a case-control study. Vascular. 2016;24:523-30.

5. Rylski B, Bavaria JE, Beyersdorf F, Branchetti E, Desai ND, Milewski RK, et al Type A aortic dissection in Marfan syndrome: extent of initial surgery determines long-term outcome. Circulation. 2014;129:1381-6.

6. Coselli JS, Green SY, Price ME, Hash JA, Ouyang Y, Volguina IV, et al. Results of open surgical repair in patients with Marfan syndrome and distal aortic dissection. Ann Thorac Surg. 2016;101:2193-201.

7. Shrestha M, Kaufeld T, Beckmann E, Fleissner F, Umminger J, Abd Alhadi F, et al Total aortic arch replacement with a novel 4-branched frozen elephant trunk prosthesis: single-center results of the first 100 patients. J Thorac Cardiovasc Surg. 2016;152:148-9.e1. 